\title{
Challenges and opportunities for future health policy
}

As this special edition of the Future Healthcare Journal illustrates, the outlook for technology and health policy is one of tremendous possibility. The fourth industrial revolution is sometimes described as the era of cyber-physical systems (following on from the eras of the microprocessor, mass production and mechanisation). For clinicians, advances in technologies like sensors, artificial intelligence, drug discovery and regenerative medicine have the potential to deliver a step-change in the efficacy and affordability of health interventions. More broadly, by harnessing large, real-time data sets, connected devices and personalised programmes, we can reframe the debate around a long-term ambition to keep people well.

The urgency of this agenda cannot be underestimated. Around the world, healthcare systems face severe pressures relating to long-term funding, demographic and lifestyle changes, sluggish productivity growth and outdated infrastructure. And yet most countries are a long way from seeking to revolutionise their approach to healthcare to meet the scale of the challenge ahead.

Here in the United Kingdom, the secretary of state's observation that he owns the world's largest collection of fax machines (and is also, thanks to Brexit, the world's largest buyer of fridges) shows in some respects how far we have to go. The gulf between the paper-based bureaucracy and the digital lives of most citizens is now decades wide, and the frustration this sometimes creates is a real risk.

In response, pulling technology leadership, policymaking and delivery together is the first step in reframing the debate about technology and the future of health policy. Yes, there is a strong progressive case for a health service that is comprehensive, universal and free at the point-of-use. And yes, there is a pragmatic case for businesses, social enterprises and not-for-profits to be partners in delivering this - as they always have been, even in single-payer systems. But, by far the biggest question is how technology can help us transcend old debates about the purpose of health policy and secure a progressive system for the long term.

As with so many other aspects of public policy, the specific technologies matter less than the shift in mindsets and approaches that the modern operating environment makes possible. For the first time it is now possible to organise services around individual needs in a way that is both highly personalised and scalable across an entire population. This in turn dramatically shifts the balance of possibilities when it comes to putting people in control and helping them to stay healthy, rather than simply accepting an ever-increasing burden that is harder and harder to cope with.

While the contexts are very different, there remains much for leaders in health policy to learn from organisations in other sectors that have been built from the ground up with the internet and technology in their DNA. In mental health, mindfulness apps and online social communities supported by clinical oversight are improving wellbeing for many. And in social care, online platforms and on-demand services can all contribute to making old-age safer, less isolated and less burdensome, with better management of supply and demand in care.

To make this shift, policymakers should prioritise reform in three key areas.

Firstly, governments need to grip the basic operational requirements for any networked organisation in the 21st century. In the healthcare arena this means things like digital patient records, online bookings and consultations, as well as the logistical infrastructure supporting things like communications and rotas. Decades of paper-based administration mean there will often be significant inertia to overcome. But, there are savings to be made, even if the up-front investment is significant, and keeping pace with the raised expectations people have in the internet age will be critical to building public and political support for reform.

Secondly, government must have the humility to accept that it cannot anticipate the needs of every patient, family and community - either now or in the decades to come. Just as Apple could never write billions of apps, it could create a platform on which others have wide latitude to innovate while remaining within strict guardrails, so policymakers need to figure out ways to promote innovation both within national healthcare systems and in partnership with academics, entrepreneurs and others.

Making this approach work means prioritising investment in the digital infrastructure required to enable this array of innovators. Leaders will need to encourage short-term experimentation and risk-taking to realise long-term progress. And pathways to procurement must be simplified to make working with government a more attractive option for the smaller companies who are often the source of so much innovation.

Thirdly, if health systems are to be less reactive and more preventative, we must build trust in the emerging technologies which might enable radically better outcomes. Take big data and artificial intelligence. This has the potential to increase the accuracy of diagnoses or to automate them altogether, to identify patterns in population-scale datasets, and to personalise treatments. But bungled implementations have caused people to question how well patient data is being protected and whether decision-makers are putting private profit before the public interest. Better civic engagement and a more honest public debate about why change is needed will be essential if we are to move forward rather than set progress back by decades.

Questions of trust and accountability will only be magnified as more radical technologies become widely accessible: gene editing, regenerative medicine, robotics and cognitive augmentation all raise profound ethical and social questions that policymakers must start to engage with now, so that we have the frameworks and systems in place to ensure our health systems can maximise the opportunities of these technologies while mitigating the very real risks they pose. 
Across all three of these domains, strong and committed political leadership will be essential - and it will be doubly difficult to achieve because the system must continue to function while also undertaking an ambitious exercise in radical transformation. But the truth is that this only heightens the urgency of bringing the right vision, mindsets and capabilities to bear on a policy challenge that is critical to the future prosperity and wellbeing of every country.

In the end, technology is driven by people, and the wave of change it is bringing about is breaking upon every country. If ridden with intelligence and purpose, it can carry us to a bright future, but if let pass, it will maroon us in a sea of irrelevance and decline. Around the world, the political parties and leaders that master this agenda and translate it into the change people want to see should own the foreseeable political future.

Tony Blair

Executive chairman of the Tony Blair Institute for Global Change and former prime minister of the United Kingdom

Chris Yiu

Head of technology and public policy, Tony Blair Institute for Global Change

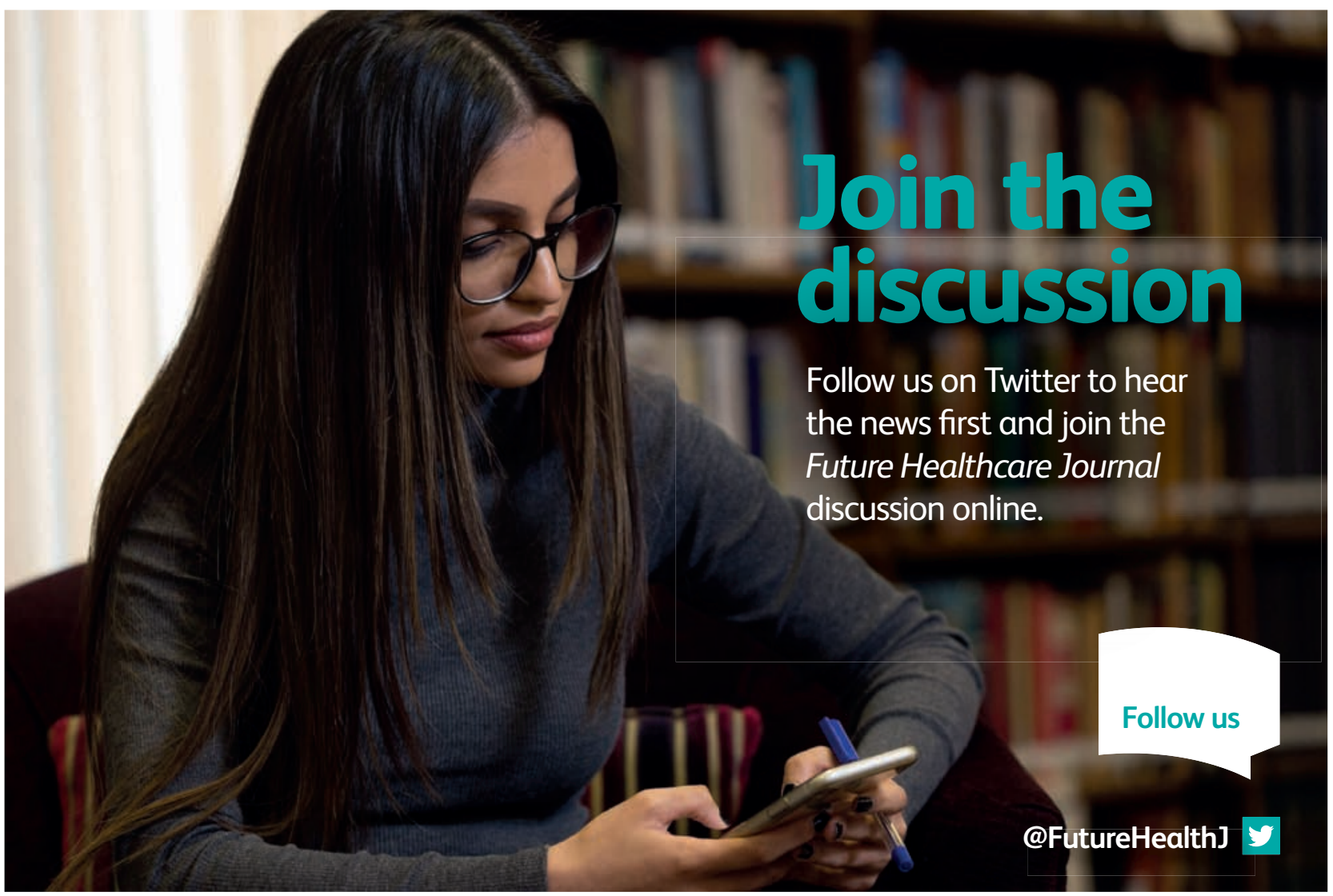

